\title{
Three stages algorithm for finding optimal solution of balanced triangular fuzzy transportation problems
}

\author{
Muhammad Sam'an \\ Postgradute Student, Faculty of Technology Management and Business, Universiti Tunn Hussein Onn, Malaysia
}

\begin{abstract}
In the literature, the fuzzy optimal solution of balanced triangular fuzzy transportation problem is negative fuzzy number. This is contrary to the constraints that must be non-negative. Therefore, the three stages algorithm is proposed to overcome this problem. The proposed algorithm consists of segregated method with segregating triangular fuzzy parameters into three crisp parameters. This method avoids the ranking technique. Next, total difference method is used to get the initial basic feasible solution (IBFS) value based on segregating triangular fuzzy parameters. While modified distribution algorithm is used to determine optimal solution based on IBFS value. In order to illustrate the proposed algorithm is given the numerical example and based on the result comparison, the proposed algorithm equality to the two existing algorithms and better than the one existing algorithm. The proposed algorithm can solve the fuzzy decision-making problems and can also be extended to an unbalanced fuzzy transportation problem.
\end{abstract}

This is an open access article under the CC BY-SA license.

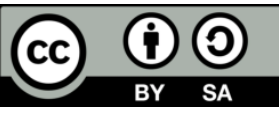

\section{Corresponding Author:}

Muhammad Sam'an,

Postgraduate Student, Faculty of Technology Management and Business,

Universiti Tunn Hussein Onn Malaysia (UTHM),

Batu Pahat, Johor, 86400, Malaysia.

Email: muhammad.92sam@gmail.com

\section{INTRODUCTION}

In real life, parameters of transportation problems (supply, demand, and transportation cost) sometimes are uncertain in values. The transportation cost, depends on fuel prices, congested routes and weather, while supply is caused by reduced quantities of raw materials, machine breakdowns and production failures. In addition, volatile market situations create uncertainty in demand. However, Zadeh [1] introduced transportation problem with the numbers of supply, demand, and transportation cost are represented by fuzzy number that is called Fuzzy Transportation Problem (FTP). The solving of FTP, many researchers changed the fuzzy parameters to crisp sets and solved by crisp transportation algorithm [2]-[12].

[13] used Yanger's ranking function to change L-R flat fuzzy parameters to crisp numbers and fuzzy classical transportation algorithm i.e. VAM (Vogel's Approximation Method), LCM (Least Cost Method) and NWC (North West Corner) to obtain L-R flat fuzzy optimal solution. [14] proposed the new ranking functionbased integral value to rank triangular fuzzy parameters and generalized fuzzy classical algorithm i.e. GVAM, GLCM and GNWC to determine a generalized triangular fuzzy optimal solution. [6] simplified the new ranking function that was proposed by [3] to rank trapezoidal fuzzy parameters and GLCM to obtain generalized trapezoidal fuzzy optimal solution. Chandran and Kandaswamy presented the score ranking method to change 
the triangular and trapezoidal fuzzy parameters and the modification of fuzzy LCM to find fuzzy optimal solution. [7] discussed the ranking method based mean of triangular fuzzy numbers and minimum demandsupply method to obtain crisp optimal solution. [9] proposed the classical ranking method and VAM-based harmonic mean method to obtain penalty value of each column and row. [15] introduced a score and accuracy function based on the Pythagorean fuzzy number and applied it on the fuzzy VAM to obtain fuzzy optimal solution. [16] presented the classical ranking function and the generalized minimum supply-demand to obtain triangular fuzzy optimal solution. [11] presented a segregated advancement scheme based minimum demandsupply method and stepping stone to obtain triangular fuzzy optimal solution.

Furthermore, the direct approach of solving FTP such as the Zero point method to obtain fuzzy optimal solution [17]. Robust ranking to rank fuzzy number and Zero suffix method to obtain the fuzzy optimal solution Fuzzy dual matrix to obtain a fuzzy optimal solution [18], [19], Improved and revised Zero point [20]-[22]. The modification of Zero-point [23]. Particle Swarm Optimization algorithm (PSO) with fuzzy constraint and conjugate constraint [24]-[27].

The use of the ranking function in the solving of FTP has a very significant impact on the resulting fuzzy optimal solution. As the results of [13] which produce a negative fuzzy optimal solution, this is contrary to the constraints that must be non-negative in the FTP model. In addition, the ranking process takes a long time to compute so that it will affect the computational performance that is not good. Therefore, in this article, we use the segregated advancement scheme approach based on the total difference method and modified distribution method to produce a fuzzy optimal solution without ranking function.

\section{THE MODELING OF BALANCED TRIANGULAR FUZZY TRANSPORTATION PROBLEM}

Based on the triangular fuzzy transportation problem with $a$ delivers and $b$ receivers. Given $\tilde{s}_{i}=$ $s_{i}^{l}, s_{i}^{m}, s_{i}^{u}$ be the triangular fuzzy supply at the $i^{t h}$ deliverer, $\tilde{d}_{j}=d_{j}^{l}, d_{j}^{m}, d_{j}^{u}$ be the triangular fuzzy demand at the $j^{\text {th }}$ receiver where $i=1,2, \ldots, a ; j=i=1,2, \ldots, b$. Let $\tilde{c}_{i j}=\left(c_{i j}^{l}, c_{i j}^{m}, c_{i j}^{u}\right)$ be the per unit triangular fuzzy transportation cost from the $i^{\text {th }}$ deliverer to the $j^{\text {th }}$ receiver and $\tilde{x}_{i j}=\left(x_{i j}^{l}, x_{i j}^{m}, x_{i j}^{u}\right)$ be the number of triangular fuzzy approximation unit to assign from the $i^{\text {th }}$ deliverer to the $j^{\text {th }}$ receiver. The modeling of balanced triangular FTP can be formulated as follows,

$$
\min Z=\sum_{i=1}^{a} \sum_{j=1}^{b} \tilde{c}_{i j} \tilde{x}_{i j}
$$

subject to

$$
\begin{array}{ll}
\sum_{j=1}^{b} \tilde{x}_{i j}=\tilde{s}_{i} & i=1,2, \ldots, a, \\
\sum_{i=1}^{a} \tilde{x}_{i j}=\tilde{d}_{j} & j=i=1,2, \ldots, b \\
\tilde{x}_{i j} \geq 0 & \forall i, j
\end{array}
$$

\section{THREE STAGES ALGORITHM FOR FINDING TRIANGULAR FUZZY OPTIMAL SOLUTION}

In this section, the three stages algorithm is proposed to find fuzzy optimal solution of a balanced triangular fuzzy transportation problem. The proposed algorithm consists of a segregated scheme to partition triangular fuzzy numbers, Total Difference Method (TDM)1 [12] to find Initial Basic Feasible Solution (IBFS) and Modified Distribution method (MODI) to obtain optimal solution based IBFS.

\section{A SEGREGATED SCHEME}

The segregated method is based on the possibility of optimized output concept of balanced triangular fuzzy transportation problem when the triangular fuzzy parameters with the corresponding demand and supply be partitioned one by one. This scheme consists of pointwise segregation of each triangular fuzzy parameters such that the first element of each triangular fuzzy parameters will be defined first segregated transportation problem is denoted $S T P^{1}$. Similarly, the second and third elements of each triangular fuzzy parameters are denoted $S T P^{3}$ and $S T P^{3}$ respectively. follows,

Using the segregated method, Eq. (1) and (2) can be transformed into the followings three STPs as 
1) $S T P^{1}$

$$
\begin{array}{ll}
\min Z_{1}^{l}=\sum_{i=1}^{a} \sum_{j=1}^{b} c_{i j}^{l} x_{i j}^{l} \\
\sum_{j=1}^{b} x_{i j}^{l}=s_{i}^{l} & i=1,2, \ldots, a, \\
\sum_{i=1}^{a} x_{i j}^{l}=d_{j}^{l} & j=i=1,2, \ldots, b \\
x_{i j}^{l} \geq 0 & \forall i, j
\end{array}
$$

2) $S T P^{2}$

$$
\begin{array}{ll}
\min Z_{2}^{m}=\sum_{i=1}^{a} \sum_{j=1}^{b} c_{i j}^{m} x_{i j}^{m} \\
\sum_{j=1}^{b} x_{i j}^{m}=s_{i}^{m} & i=1,2, \ldots, a, \\
\sum_{i=1}^{a} x_{i j}^{m}=d_{j}^{m} & j=i=1,2, \ldots, b \\
x_{i j}^{m} \geq 0 & \forall i, j
\end{array}
$$

3) $\operatorname{STP}^{3}$

$$
\begin{array}{ll}
\min Z_{3}^{u}=\sum_{i=1}^{a} \sum_{j=1}^{b} c_{i j}^{u} x_{i j}^{u} \\
\sum_{j=1}^{b} x_{i j}^{u}=s_{i}^{u} & i=1,2, \ldots, a, \\
\sum_{i=1}^{a} x_{i j}^{u}=d_{j}^{u} & j=i=1,2, \ldots, b \\
x_{i j}^{u} \geq 0 & \forall i, j
\end{array}
$$

\subsection{Total Difference Method 1}

For a crisp balanced transportation problem with transportation cost matrix of order $(a, b)$ having supply $s_{i}^{q}, i=1,2, \ldots, a$; demand $d_{j}^{q}, i=j=1,2, \ldots, b$ and the corresponding transportation $\operatorname{cost} c_{i j}^{q}, q=$ $l, m, u$. In detail, TDM 1 algorithm shown in Algorithm 1 shown in Figure 1.

\subsection{Modified Distribution Method}

MODI to obtain the optimal solution of balanced triangular fuzzy transportation problem Algorithm 2 shown in Figure 2.

\subsection{Three Stages Algorithm}

Three stages algorithm to obtain the optimal solution of balanced triangular fuzzy transportation problem shown Algorithm 3 shown in Figure 3. 


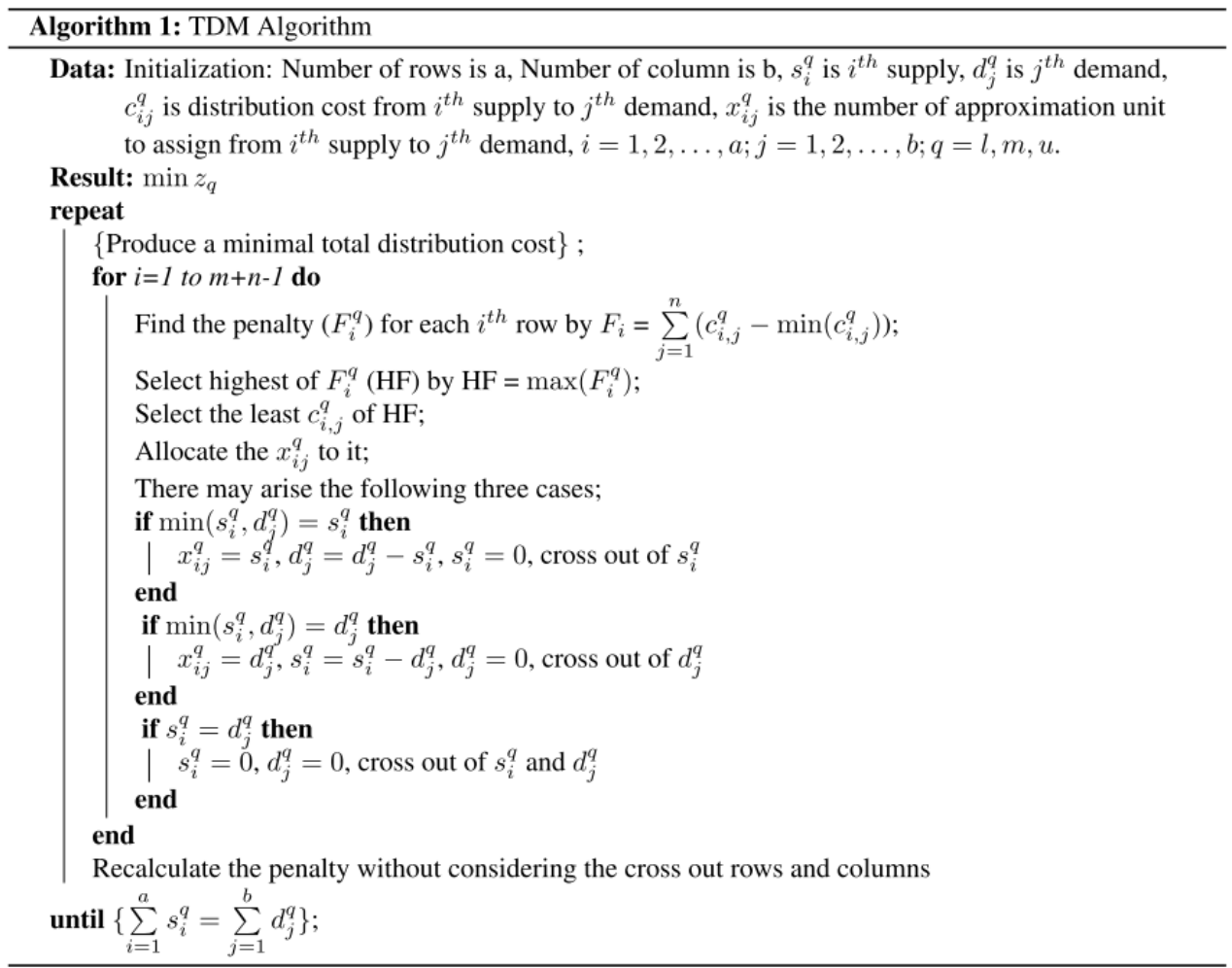

Figure 1. TDM 1 algorithm

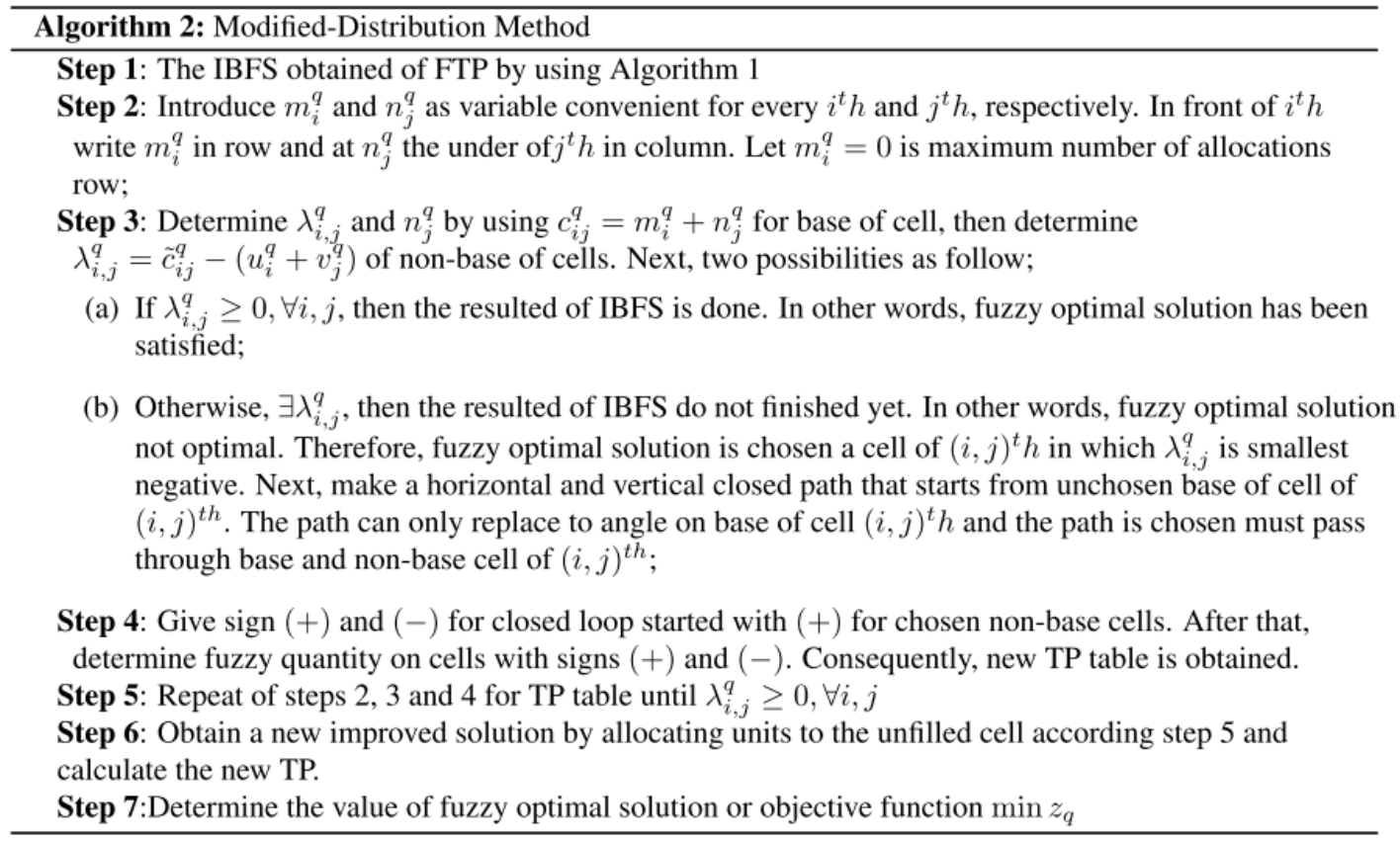

Figure 2. MODI algorithm 


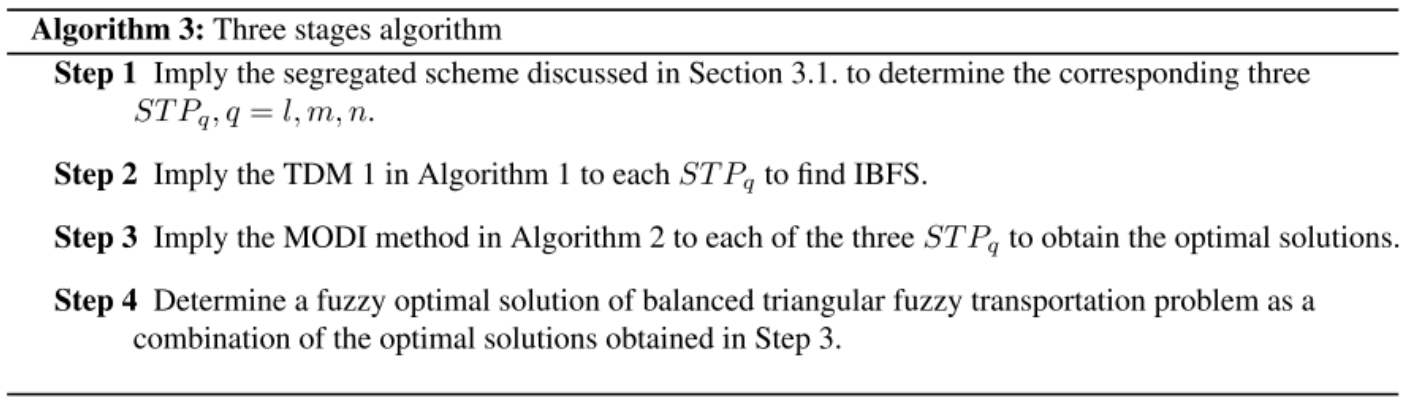

Figure 3. Three stages algorithm

\section{NUMERICAL ILLUSTRATION}

In this section, we illustrate the proposed method by using numerical example is adapted from Ebrahimnejad [28] in which a trading company wants to optimize the transportation cost of products.

Example 1 A leading trading factory wants to obtain the fuzzy number of the commodity that should be distributed from each warehouse to each object such that the total fuzzy transportation cost is at a minimum. The factory has two production houses and three distribution centers. The parameters of transportation are fuzzy numbers that are represented by triangular fuzzy numbers because of disinformation real-life conditions. The summary of fuzzy parameters i.e. supply, demand and transportation cost (dollar) are shown in Table 1 with $\mathrm{l}$ as the least amount value, $\mathrm{m}$ as the most possible value and $\mathrm{u}$ as the greatest amount value.

Table 1. Data of example 1 (in U.S. dollar)

\begin{tabular}{|c|c|c|c|c|}
\hline \multirow{2}{*}{ Parameters } & & \multicolumn{3}{|c|}{ Triangular fuzzy numbers } \\
\hline & & Least amount value (1) & Most possible value (m) & Greatest amount value (n) \\
\hline \multirow{6}{*}{ Transportation cost $(\$)$} & $c_{11}$ & 15 & 25 & 35 \\
\hline & $c_{12}$ & 55 & 65 & 85 \\
\hline & $c_{13}$ & 85 & 95 & 105 \\
\hline & $c_{21}$ & 65 & 75 & 85 \\
\hline & $c_{22}$ & 80 & 90 & 110 \\
\hline & $c_{23}$ & 30 & 40 & 50 \\
\hline \multirow{2}{*}{ Supply in unit } & $s_{1}$ & 75 & 95 & 125 \\
\hline & $s_{2}$ & 45 & 65 & 95 \\
\hline \multirow{3}{*}{ Demand in unit } & $d_{1}$ & 35 & 45 & 65 \\
\hline & $d_{2}$ & 25 & 35 & 45 \\
\hline & $d_{3}$ & 60 & 80 & 110 \\
\hline
\end{tabular}

Solution: After implementing the segregated scheme discussed in Section 5.1, three crisp transportation problems i.e. ST $P^{1}, S T P^{2}, S T P^{3}$ are resulted as follows:

1) for $S T P^{1}$ by using Eq. (3) and (4)

$$
\min Z_{1}^{l}=15 x_{11}^{l}+55 x_{12}^{l}+85 x_{13}^{l}+65 x_{21}^{l}+80 x_{22}^{l}+30 x_{23}^{l}
$$

subject to

2) for $S T P^{2}$ by using Eq. (5) and (6)

$$
\begin{aligned}
& x_{11}^{l}+x_{12}^{l}+x_{13}^{l}=75 \\
& x_{21}^{l}+x_{22}^{l}+x_{23}^{l}=45 \\
& x_{11}^{l}+x_{21}^{l}=35 \\
& x_{12}^{l}+x_{22}^{l}=25 \\
& x_{13}^{l}+x_{23}^{l}=25
\end{aligned}
$$

$$
\min Z_{2}^{m}=25 x_{11}^{m}+65 x_{12}^{m}+95 x_{13}^{m}+75 x_{21}^{m}+90 x_{22}^{m}+40 x_{23}^{m}
$$


subject to

$$
\begin{aligned}
& x_{11}^{m}+x_{12}^{m}+x_{13}^{m}=95 \\
& x_{21}^{m}+x_{22}^{m}+x_{23}^{m}=65 \\
& x_{11}^{m}+x_{21}^{m}=45 \\
& x_{12}^{m}+x_{22}^{m}=35 \\
& x_{13}^{m}+x_{23}^{m}=805
\end{aligned}
$$

3) for $S T P^{3}$ by using Eq. (7) and (8)

$$
\min Z_{3}^{u}=35 x_{11}^{u}+85 x_{12}^{u}+105 x_{13}^{u}+85 x_{21}^{u}+110 x_{22}^{u}+50 x_{23}^{u}
$$

subject to

$$
\begin{aligned}
& x_{11}^{u}+x_{12}^{u}+x_{13}^{u}=125 \\
& x_{21}^{u}+x_{22}^{u}+x_{23}^{u}=95 \\
& x_{11}^{u}+x_{21}^{u}=65 \\
& x_{12}^{u}+x_{22}^{u}=45 \\
& x_{13}^{u}+x_{23}^{u}=110
\end{aligned}
$$

After implementing the TDM 1 in Algorithm 5.2, the penalty value resulted

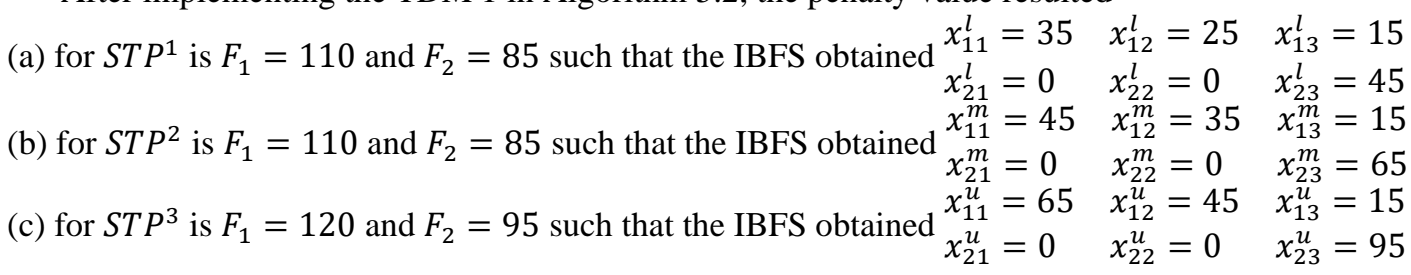

After implementing the MODI method shown in Algorithm 2() @ is used to obtain optimal solution based on the IBFS value of all three $S T P_{q}, q=l, m, n$ and resulted in minimal transportation cost for $S T P_{l}=$ 4535, $S T P_{m}=7425$ and $S T P_{u}=12425$.

After combining the minimal transportation cost of all three $S T P_{q}$, so that the fuzzy optimal solution of balanced triangular fuzzy optimal solution is $(4525,7425,1245)$ where represents that the minimal transportation cost most likely will be $\$ 7,425$ but certainly not less than $\$ 4,525$. Meanwhile, if things are not in favor of decision-maker, it could be as high as $\$ 12,425$.

\section{CONCLUSION}

In this article, the three stages algorithm consist of a segregated method, total difference method 1 and modified distribution method is proposed to optimize of balanced triangular fuzzy transportation problem. A segregated method scheme and TDM 1 are used to get IBFS value, meanwhile, the MODI method to obtain optimal solution based IBFS value. The numerical example is given to illustrate the justification of proposed algorithm. The Comparative study of results with the literature journal shows that the fuzzy optimal solution that is resulted by proposed algorithm equivalent to those by [13] and [11] and better than those by [2]. All the acquired allocations by proposed algorithm there are non-negative triangular fuzzy numbers whereas one allocation by [2] is negative and violates the basic rule of trading.

\section{REFERENCES}

[1] L. A. Zadeh, "Fuzzy sets," Inf. Control, vol. 8, no. 3, pp. 338-353, 1965, doi: 10.1016/S00199958(65)90241-X.

[2] A. Kumar, J. Kaur, and P. Singh, "A new method for solving fully fuzzy linear programming problems," Appl. Math. Model., vol. 35, no. 2, pp. 817-823, 2011, doi: 10.1016/j.apm.2010.07.037.

[3] S. Chandran and G. Kandaswamy, "A fuzzy approach to transport optimization problem," Optim. Eng., vol. 17, no. 4, pp. 965-980, 2016, doi: 10.1007/s11081-012-9202-6.

[4] G. De França Aguiar, B. De Cássia Xavier Cassins Aguiar, and V. E. Wilhelm, "New methodology to find initial solution for transportation problems: A case study with fuzzy parameters," Appl. Math. Sci., 
vol. 9, no. 17-20, pp. 915-927, 2015, doi: 10.12988/ams.2015.4121018.

[5] A. Kumar and P. Singh, "A new method for solving fully fuzzy linear programming problems," Ann. Fuzzy Math. Inf., vol. 3, pp. 103-118, 2012.

[6] A. Ebrahimnejad, "A simplified new approach for solving fuzzy transportation problems with generalized trapezoidal fuzzy numbers," Appl. Soft Comput. J., vol. 19, pp. 171-176, 2014, doi: 10.1016/j.asoc.2014.01.041.

[7] N. Mathur, P. K. Srivastava, and A. Paul, "Trapezoidal fuzzy model to optimize transportation problem," Int. J. Model. Simulation, Sci. Comput., vol. 7, no. 3, 2016, doi: 10.1142/S1793962316500288.

[8] D. Hunwisai and P. Kumam, "A method for solving a fuzzy transportation problem via Robust ranking technique and ATM," Cogent Math., vol. 4, no. 1, 2017, doi: 10.1080/23311835.2017.1283730.

[9] K. Balasubramanian and S. Subramanian, "Optimal solution of fuzzy transportation problems using ranking function," Int. J. Mech. Prod. Eng. Res. Dev., vol. 8, no. 4, pp. 551-558, 2018, doi: 10.24247/ijmperdaug201856.

[10] D. Chakraborty, D. K. Jana, and T. K. Roy, "A new approach to solve fully fuzzy transportation problem using triangular fuzzy number," Int. J. Oper. Res., vol. 26, no. 2, pp. 153-179, 2016, doi: 10.1504/IJOR.2016.076299.

[11] R. K. Saini, A. Sangal, and O. Prakash, "Fuzzy transportation problem with generalized triangulartrapezoidal fuzzy number," in Advances in Intelligent Systems and Computing, 2018, vol. 583, pp. 723-734, doi: 10.1007/978-981-10-5687-1_64.

[12] D. Rani and T. R. Gulati, "A new approach to solve unbalanced transportation problems in imprecise environment," J. Transp. Secur., vol. 7, no. 3, pp. 277-287, 2014, doi: 10.1007/s12198-014-0143-5.

[13] A. Kumar and A. Kaur, "Application of Classical Transportation Methods to Find the Fuzzy Optimal Solution of Fuzzy Transportation Problems," Fuzzy Inf. Eng., vol. 3, no. 1, pp. 81-99, 2011, doi: 10.1007/s12543-011-0068-7.

[14] A. Kaur and A. Kumar, "A new approach for solving fuzzy transportation problems using generalized trapezoidal fuzzy numbers," Appl. Soft Comput. J., vol. 12, no. 3, pp. 1201-1213, 2012, doi: 10.1016/j.asoc.2011.10.014.

[15] R. Kumar, S. A. Edalatpanah, S. Jha, and R. Singh, "A Pythagorean fuzzy approach to the transportation problem," Complex Intell. Syst., vol. 5, no. 2, pp. 255-263, 2019, doi: 10.1007/s40747019-0108-1.

[16] P. K. Srivastava and D. C. S. Bisht, "A Segregated Advancement in the Solution of Triangular Fuzzy Transportation Problems," Am. J. Math. Manag. Sci., pp. 1-11, 2020, doi: 10.1080/01966324.2020.1854137.

[17] P. Pandian and G. Natarajan, "A new algorithm for finding a fuzzy optimal solution for fuzzy transportation problems," Appl. Math. Sci., vol. 4, no. 1-4, pp. 79-90, 2010, doi: 10.1504/IJMMNO.2021.111715.

[18] A. Edward Samuel and M. Venkatachalapathy, "IZPM for unbalanced fuzzy transportation problems," Int. J. Pure Appl. Math., 2013, doi: 10.12732/ijpam.v86i4.8.

[19] K. Selvakumari and S. Sathya Geetha, "A new approach for solving intuitionistic fuzzy transportation problem," J. Adv. Res. Dyn. Control Syst., vol. 12, no. 5 Special Issue, pp. 956-963, 2020, doi: 10.5373/JARDCS/V12SP5/20201841.

[20] A. Edward Samuel and M. Venkatachalapathy, "Improved zero point method for solving fuzzy transportation problems using ranking function," Far East J. Math. Sci., vol. 75, no. 1, pp. 85-100, 2013.

[21] A. Edward Samuel, "Improved zero point method (IZPM) for the transportation problems," Appl. Math. Sci., vol. 6, no. 109-112, pp. 5421-5426, 2012.

[22] T. Karthy and K. Ganesan, "Improved zero point method for the fuzzy optimal solution to fuzzy transportation problems," Glob. J. Pure Appl. Math., vol. 12, no. 1, pp. 255-260, 2016.

[23] A. Akilbasha, G. Natarajan, and P. Pandian, "A new approach for solving transportation problems in fuzzy nature," Int. J. Appl. Eng. Res., vol. 11, no. 1, pp. 498-502, 2016.

[24] A. Baykasoğlu and K. Subulan, "A direct solution approach based on constrained fuzzy arithmetic and metaheuristic for fuzzy transportation problems," Soft Comput., vol. 23, no. 5, pp. 1667-1698, 2019, doi: 10.1007/s00500-017-2890-2.

[25] M. A. Imron and B. Prasetyo, "Improving algorithm accuracy K-Nearest Neighbor using Z-Score Normalization and Particle Swarm Optimization to predict customer churn," J. Soft Comput. Explor., vol. 1, no.1, pp. 56-62, 2020.

[26] R. H. Saputra and B. Prasetyo, "Improve the accuracy of c4.5 algorithm using particle swarm optimization (pso) feature selection and bagging technique in breast cancer diagnosis," J. Soft Comput. Explor., vol. 1, no.1, pp. 47-55, 2020. 
[27] D. Aprilianto, "SVM optimization with correlation feature selection based binary particle swarm optimization for diagnosis of chronic kidney disease," J. Soft Comput. Explor., vol. 1, no.1, pp. 2431, 2020.

[28] A. Ebrahimnejad, "An improved approach for solving fuzzy transportation problem with triangular fuzzy numbers,” J. Intell. Fuzzy Syst., vol. 29, no. 2, pp. 963-974, 2015, doi: 10.3233/IFS-151625. 